\title{
Correspondence
}

\section{Is nail trephining safe?}

\section{Sir,}

Injury to the fingertip or toe often results in a subungual haematoma. Some of these patients, and we do not know the exact proportion, will have sustained a fracture of the terminal phalanx.

If the haematoma is painful, the usual treatment is to trephine the nail. ${ }^{1}$ Presumably this converts a closed fracture (if present) to a compound one. This being the case, it seems possible that trephining (or not trephining) may increase the potential for osteomyelitis. Local inquiries suggest that such a hazard is purely theoreticalhas anyone seen this complication?

We wonder if it is safer to obtain an $x$-ray and avoid trephining a subungual haematoma if a fracture is present.

\section{Reference}

1 Illingworth C M. The diagnosis and primary care of accidents and emergencies in children. $A$ manual for the casualty officer and the family doctor. Oxford: Blackwell Scientific Publications, 1978: 79.

\section{G H HuTCHInson AND T J DAVID Booth Hall Children's Hospital, Charlestown Road, Blackley, Manchester M9 $2 A A$}

\section{Cimetidine and peptic ulceration in \\ a Meckel's diverticulum}

\section{Sir,}

Cimetidine is an effective agent in duodenal ulceration, ${ }^{1}$ and has been used in the management of gastrointestinal bleeding particularly that due to gastric ulceration. ${ }^{2}$ It has also been used in the treatment of haemorrhage from a Meckel's diverticulum when there is a contraindication to surgery. ${ }^{3}$ We report the results of treatment with cimetidine in a boy with a Meckel's diverticulum and Duchenne muscular dystrophy.

A 12-year-old boy with Duchenne muscular dystrophy was admitted with a 24-hour history of melaena and fresh rectal bleeding. There had been several episodes of nonspecific abdominal pain. Barium studies were negative but a technetium scan showed an appearance that suggested a Meckel's diverticulum. In view of his condition, treatment with cimetidine $100 \mathrm{mg}$ 6-hourly was started. The boy remained asymptomatic for 3 months and then, while still on treatment, presented with acute generalised peritonitis. At laparotomy a perforated Meckel's diverticulum was removed, histology of which showed ulcerated epithelium of gastric type. Postoperative recovery was uneventful.
Although successful treatment with cimetidine of bleeding Meckel's diverticulum has been recorded ${ }^{3}$ this experience suggests that there are major shortcomings in this line of management. Although there may be fairly adequate control of pain and even of bleeding, it should probably only be regarded as a short-term rather than long-term treatment. Clearly perforation of a Meckel's diverticulum can occur while the patient is on $\mathrm{H}_{2}$ antagonist therapy. We confirm that surgery is the treatment of choice for this condition.

\section{References}

1 Gray G R, McKenzie I, Smith I S, Crean G P, Gillespie G. Oral cimetidine in severe duodenal ulceration. A doubleblind controlled trial. Lancet $1977 ; 1$ : 4-7.

2 Dykes P W, Hoare A M, Hawkins C F, Kang J Y. The treatment of upper gastrointestinal haemorrhage with cimetidine. In: Wastell C, Lance P, eds. Proceedings of the Westminster Hospital Symposium on cimetidine. Edinburgh: Churchill Livingstone, 1978: 173-9.

3 Kirkpatrick R A. Letter: Cimetidine and Meckel's diverticulum. Ann Intern Med 1978; 88 : 846-7.

P E MINCHOM, M H WHEELER, AND J RSIBERT Department of Paediatrics and Surgery, Llandough Hospital, Penarth, Glamorgan CF6 $1 X X$

\section{Comparing methods of assessing gestational age}

Sir, One of the more common types of medical investigation is to compare two methods of measuring something. Although this is one of the most straightforward of statistical problems it seems to produce more than its fair share of incorrect analyses.

Dr Cater ${ }^{1}$ has compared gestational age derived from a total maturity score (TMS) with gestational age calculated from the date of the last menstrual period (LMP). The two gestational ages were compared for 76 low birthweight (LBW) babies and 80 'matched controls'. (Why there are 80 controls and what they were matched for we are not told.) The LBW babies were divided into term and preterm groups, presumably on the basis of the LMP.

For each of the three groups - controls, preterm LBW, and term LBW-an unidentified statistical test was performed to compare the mean gestation by each method. If this had been done using a paired $t$ test (or a Wilcoxon test if the within-subject differences were nonGaussian) this would have been the correct way of 
determining if one method was biased compared with the other. However, this does not answer the more important question, 'Does the TMS-derived gestational age agree sufficiently well with the LMP-derived age for individual babies?' To answer this question it is necessary to consider the distribution of the within-person differences between the ages derived by each method (see, for example, Wu et al. ${ }^{2}$ ).

A separate criticism of this paper concerns the division of LBW babies into term and preterm groups. While it may be of interest to see if the results are different for the two groups, the only relevant analysis is that for all LBW babies together. The whole point of the exercise is to see if the TMS method can accurately assess gestational age, and distinguishing between LBW babies of different gestations is an important aspect which Cater's approach cannot fully consider. Even if the statistical approach were valid, a conclusion that the method works for term babies is of limited value if you do not know exactly which babies these are.

I recently made similar remarks ${ }^{3}$ about a paper by Serfontein and Jaroszewicz ${ }^{4}$ in which two other methods of assessing gestational age (Dubowitz's and Robinson's) were compared. Serfontein and Jaroszewicz ${ }^{4}$ had used correlation - a method which Dr Cater rightly rejects, but for the wrong reason. Their reply to my previous letter dismissed my criticisms as minor. They were however, fundamental, as are my criticisms of Dr Cater's paper. It is not statistical quibbling to raise these points. Each type of analysis tests a different hypothesis; if you choose an incorrect analysis you will test an inappropriate hypothesis.

As in the earlier paper, Dr Cater's conclusion that two different methods are comparable is totally unjustified. In both cases the authors have collected the correct data to enable them to answer their question, but have used incorrect analyses, and in both the data they present suggest that agreement may not be particularly good.

A minimum requirement for the comparison of methods is a scatter diagram relating the methods and indicating the line of equality, together with an analysis of the within-person differences between methods.

\section{References}

1 Cater J I. Confirmation of gestational age by external physical characteristics (total maturity score). Arch Dis Child 1979; 54: 794-5.

2 Wu G T, Twomey S L, Thiers R E. Statistical evaluation of method-comparison data. Clin Chem 1975; 21 : 315-20.

3 Altman D G. Letter: Estimation of gestational age at birth-comparison of two methods. Arch Dis Child 1979; 54: 242-3.

4 Serfontein G L, Jaroszewicz A M. Estimation of gestational age at birth. Comparison of two methods. Arch Dis Child 1978; 53: 509-11.

D G Altman

Clinical Research Centre, Division of Computing and Statistics, Watford Road, Harrow, Middlesex $\mathrm{HAl}$ 3UJ
Dr Cater comments :

I am grateful to Mr Altman for his interest and comments. The infants studied were a sample from a larger survey of LBW babies and their controls were matched for paternal occupation, ordinal position in the family, maternal height, smoking, and sex of the baby. Those of certain gestation were selected as controls and were therefore not strictly matched.

Mr Altman is right in saying that the paired $t$ test will not prove the equivalent of TMS and LMP methods. It is however a necessary if not sufficient condition if the two methods are equivalent for the means to be the same. It is precisely for this reason that the further analysis of subgroups is relevant since the equivalent of means for these subgroups should also be demonstrated.

I cannot agree that the only relevant analysis is to consider all the LBW babies together. Detection of bias in the TMS for particular subgroups may suggest possible recalibration to improve the scoring system.

As clinical methods for corroborating maternal menstrual data in neonatal work become more widespread, it is important to be aware of both the clinical and the statistical limitations of the available scoring methods. Further study of this is proceeding.

JOHN I CATER

Department of Child Health, Ninewells Hospital and Medical School, Dundee DD1 9SY Angus

\section{Exchange transfusion in newborn infants}

Sir,

We were interested to read the report by Pelet $^{1}$ of the effect of exchange transfusion on opsonisation and granulocyte function. While he clearly shows that exchange transfusion increases the capacity of serum to promote phagocytosis of heat-killed bakers' yeast, this may not be the same phenomenon as correction of the isolated defect of yeast opsonisation by plasma as described by Soothill and Harvey. ${ }^{2}$ Newborn infants have a number of immunodeficiencies and one in particular, namely deficiency of factor B of the alternative pathway of complement, ${ }^{3}$ is likely to be important in the pathogenesis of defective yeast opsonisation. We have shown that children with fulminant hepatic failure (FHF) not only have defective yeast opsonisation, but also profound defects of functional activity of both $\mathrm{C} 3$ and factor B (V F Larcher, $\mathbf{R} \mathbf{J}$ Wyke, A P Mowat, $\mathbf{R}$ Williams, in preparation). The addition of subopsonising amounts of normal sera to FHF sera restores yeast opsonisation, as it does in children with primary yeast opsonisation deficiency, but addition of factor B-depleted sera, even in equal volumes, did not provide more than $50 \%$ restoration of opsonising activity. On the other hand, the opsonising capacity of sera from patients with primary isolated yeast opsonisation deficiency can be fully restored by the addition of factor B-depleted sera. 